\title{
Bounce and cyclic cosmology in extended nonlinear massive gravity
}

\author{
Yi-Fu Cai ${ }^{a, b}$ Caixia Gao ${ }^{c}$ Emmanuel N. Saridakis $^{d, e, f}$ \\ ${ }^{a}$ Department of Physics, Arizona State University, Tempe, AZ 85287, USA \\ ${ }^{b}$ Department of Physics, McGill University, Montréal, QC, H3A 2T8, Canada \\ ${ }^{c}$ Department of Physics, University of Mississippi, Oxford, MS 386r7, USA \\ ${ }^{d}$ Physics Division, National Technical University of Athens, 15780 Zografou Campus, Athens, \\ Greece \\ ${ }^{e}$ CASPER, Physics Department, Baylor University, Waco, TX 76798-7310, USA \\ ${ }^{f}$ Institut d'Astrophysique de Paris, UMR 7095-CNRS, Université Pierre 6 Marie Curie, 98bis \\ boulevard Arago, 75014 Paris, France \\ E-mail: ycai21@asu.edu, cgao1@go.olemiss.edu, \\ Emmanuel_Saridakis@baylor.edu
}

ABSTRACT: We investigate non-singular bounce and cyclic cosmological evolutions in a universe governed by the extended nonlinear massive gravity, in which the graviton mass is promoted to a scalar-field potential. The extra freedom of the theory can lead to certain energy conditions violations and drive cyclicity with two different mechanisms: either with a suitably chosen scalar-field potential under a given Stückelberg-scalar function, or with a suitably chosen Stückelberg-scalar function under a given scalar-field potential. Our analysis shows that extended nonlinear massive gravity can alter significantly the evolution of the universe at both early and late times.

KEYwords: Modified Gravity, massive gravity, bounce, cyclic cosmology 


\section{Contents}

1 Introduction 1

$\begin{array}{lll}2 & \text { Cosmology in extended nonlinear massive gravity } & 2\end{array}$

3 Bouncing and Cyclic solutions 4

3.1 Known $b(\tau) \quad 5$

3.2 Known $W(\psi) \quad 9$

4 Discussion $\quad 12$

\section{Introduction}

The question on whether there exits a consistent covariant theory for massive gravity, where the graviton acquires a mass and leads to a modification of General Relativity at large distances, was initiated by Fierz and Pauli a long time ago [1]. However, it was observed that the nonlinear terms required by massive gravity [2], which can give rise to continuity of observables $[3,4]$, lead inevitably to the existence of the Boulware-Deser (BD) ghost [5], and thus make the theory unstable.

Although for the subsequent decades it was believed that there is no consistent way to construct a massive gravity free of ghosts, a nonlinear extension of massive gravity was constructed recently by de Rham, Gabadadze and Tolley $[6,7]$. In this model, the BD ghost can be removed in the decoupling limit to all orders in perturbation theory through a systematic construction of a covariant nonlinear action (see [8] for a review). Although it is still controversial to prove the absence of $\mathrm{BD}$ ghost at the non-perturbative level, the theoretical and phenomenological advantages led to a wide investigation of this theory [9-52].

Despite the successes of nonlinear massive gravity, it was also noticed that certain cosmological instabilities still exist in the case where the physical and the fiducial metrics have simple homogeneous and isotropic forms [42]. This behavior motivated researches towards extensions of nonlinear gravity models, namely the construction of nonlinear massive gravity with less symmetric metrics [13, 43]. However, in [53] a different approach was followed, and nonlinear massive gravity was extended allowing for the graviton mass to vary. This could be realized by introducing an additional scalar field, which coupling to the graviton potentials produces an effective, varying, graviton mass. Moreover, this extension provides a natural way to modify General Relativity not only in the IR but also in the UV, since the graviton mass can be evolved into a large value at the early universe [54]. Therefore, it is interesting to study the cosmological implications of this scenario at early times, and this is a main topic of the present work. 
On the other hand, it is well known that cosmological evolution governed by standard Einstein gravity usually suffers from the problem of initial singularity if Null Energy Condition (NEC) is preserved [55]. A potential solution to the cosmological singularity problem may be provided by non-singular bouncing cosmologies [56-58]. Such scenarios have been constructed within various approaches to modified gravity [59-77]. Generally, a non-singular bouncing model can be acquired by using NEC violating matter [78-84], by making use of various mechanisms [85-92]. Note that in the case of a positive curvature a generic bounce can be obtained by violating Strong Energy Condition (SEC) only [93], however the singularity reappears in the fact that the number of regular bounces is finite [94]. Furthermore, the perturbation theory of non-singular bounce cosmology and its relation to observables was extensively studied in the literature [82-84, 95-100]. The extension of all the above bouncing scenarios is the old idea of cyclic cosmology [101], in which the universe presents a periodic sequence of contractions and expansions. Cyclic cosmology has attracted a significant interest the last years [102] since it brings different insights for the origin of the observable universe [103-114] (see [115, 116] for recent reviews).

In the present work we are interested in constructing scenarios of cyclic cosmology in a universe governed by the extended, varying-mass, nonlinear massive gravity. Particularly, we first determine the Stückelberg-scalar function and we suitably reconstruct the form of the potential of the scalar field which leads to a cyclic universe. Alternatively, we first determine the scalar potential and we reconstruct the Stückelberg-scalar function in order to obtain cyclicity. Interestingly enough cyclicity is easily acquired in this framework, since extended nonlinear massive gravity can violate certain energy conditions and therefore has fruitful implications to physics of the universe at both early and late times.

This paper is organized as follows. In Section 2 we briefly review the cosmological equations under the extended scenario of nonlinear massive gravity with a scalar field being introduced to evolve the graviton mass. In section 3 we construct scenarios of bouncing and cyclic universe. In particular, in subsection 3.1 we start with a given Stückelberg-scalar function and we reconstruct the scalar potential; while in 3.2 we start from a given scalar potential and we determine the corresponding Stückelberg-scalar function that leads to cyclicity. Finally, section 4 is devoted to the summary of our results.

\section{Cosmology in extended nonlinear massive gravity}

In this section we briefly review cosmology in extended nonlinear massive gravity [53, 54]. In such a scenario the graviton mass is upgraded into an evolving function depending on a cosmic scalar field, of which a canonical kinetic term and a standard potential are added in the action too. In this construction, the total action is written as

$$
S=\int d^{4} x \sqrt{-g}\left[\frac{M_{P}^{2}}{2} R+V(\psi)\left(U_{2}+\alpha_{3} U_{3}+\alpha_{4} U_{4}\right)-\frac{1}{2} \partial_{\mu} \psi \partial^{\mu} \psi-W(\psi)\right]+S_{m},
$$

where $M_{P}$ is the reduced Planck mass, $R$ is the Ricci scalar, and $\psi$ is the newly introduced scalar field with $W(\psi)$ its usual potential and $V(\psi)$ an additional potential coupling to the 
graviton potentials. These graviton potentials are given by,

$$
U_{2}=\mathcal{K}_{[\mu}^{\mu} \mathcal{K}_{\nu]}^{\nu}, \quad U_{3}=\mathcal{K}_{[\mu}^{\mu} \mathcal{K}_{\nu}^{\nu} \mathcal{K}_{\rho]}^{\rho}, \quad U_{4}=\mathcal{K}_{[\mu}^{\mu} \mathcal{K}_{\nu}^{\nu} \mathcal{K}_{\rho}^{\rho} \mathcal{K}_{\sigma]}^{\sigma}
$$

with

$$
\mathcal{K}_{\nu}^{\mu} \equiv \delta_{\nu}^{\mu}-\sqrt{g^{\mu \rho} f_{A B} \partial_{\rho} \phi^{A} \partial_{\nu} \phi^{B}}, \quad \mathcal{K}_{[\mu}^{\mu} \mathcal{K}_{\nu]}^{\nu} \equiv \frac{1}{2}\left(\mathcal{K}_{\mu}^{\mu} \mathcal{K}_{\nu}^{\nu}-\mathcal{K}_{\nu}^{\mu} \mathcal{K}_{\mu}^{\nu}\right)
$$

and similarly for the other antisymmetric expressions. Moreover, $\alpha_{3}$ and $\alpha_{4}$ are dimensionless parameters. Additionally, $f_{A B}$ is a fiducial metric, and the four-form fields $\phi^{A}(x)$ are the Stückelberg scalars introduced to restore general covariance [117]. As it was shown in [53] the above scenario is still free of the the BD ghost. Finally, in the action (2.1) we can take into account the standard matter action $S_{m}$, minimally-coupled to the dynamical metric, corresponding to energy density $\rho_{m}$ and pressure $p_{m}$.

In order to derive explicitly the cosmological equations, we need to impose certain ansatzes for the two metrics. For the physical metric we consider a flat FriedmannRobertson-Walker (FRW) form (one can also straightforwardly investigate the non-flat case [53]):

$$
d^{2} s=-N(\tau)^{2} d \tau^{2}+a(\tau)^{2} \delta_{i j} d x^{i} d x^{j}
$$

with $N(\tau)$ the lapse function and $a(\tau)$ the scale factor, and for simplicity for the Stückelberg fields we choose the forms as follows,

$$
\phi^{0}=b(\tau), \quad \phi^{i}=a_{r e f} x^{i},
$$

with $a_{\text {ref }}$ a constant positive coefficient. Note that although in standard massive gravity such a choice for the dynamical metric cannot be accompanied by a simple choice for the fiducial one, in the scenario at hand the extra freedom allows for a simple Minkowski ansatz for the fiducial metric

$$
f_{A B}=\eta_{A B} .
$$

Variations of the action with respect to $N$ and $a$ give rise to the following Friedmann equations

$$
\begin{aligned}
3 M_{P}^{2} H^{2} & =\rho_{M G}+\rho_{m}, \\
-2 M_{P}^{2} \dot{H} & =\rho_{M G}+p_{M G}+\rho_{m}+p_{m},
\end{aligned}
$$

where we have defined the Hubble parameter $H=\dot{a} / a$, with $\dot{a}=d a /(N d \tau)$. In the end we adopt $N=1$ for convenience. In the above expressions we have defined the energy density and pressure arising from the modified gravitational sector as

$$
\begin{aligned}
\rho_{M G} & =\frac{1}{2} \dot{\psi}^{2}+W(\psi)+V(\psi)\left(\frac{a_{r e f}}{a}-1\right)\left[f_{3}(a)+f_{1}(a)\right] \\
p_{M G} & =\frac{1}{2} \dot{\psi}^{2}-W(\psi)-V(\psi) f_{4}(a)-V(\psi) \dot{b} f_{1}(a)
\end{aligned}
$$


with

$$
\begin{aligned}
& f_{1}(a)=3-\frac{2 a_{r e f}}{a}+\alpha_{3}\left(3-\frac{a_{r e f}}{a}\right)\left(1-\frac{a_{r e f}}{a}\right)+\alpha_{4}\left(1-\frac{a_{r e f}}{a}\right)^{2} \\
& f_{2}(a)=1-\frac{a_{r e f}}{a}+\alpha_{3}\left(1-\frac{a_{r e f}}{a}\right)^{2}+\frac{\alpha_{4}}{3}\left(1-\frac{a_{r e f}}{a}\right)^{3} \\
& f_{3}(a)=3-\frac{a_{r e f}}{a}+\alpha_{3}\left(1-\frac{a_{r e f}}{a}\right) \\
& f_{4}(a)=-\left[6-\frac{6 a_{r e f}}{a}+\left(\frac{a_{r e f}}{a}\right)^{2}+\alpha_{3}\left(1-\frac{a_{r e f}}{a}\right)\left(4-\frac{2 a_{r e f}}{a}\right)+\alpha_{4}\left(1-\frac{a_{r e f}}{a}\right)^{2}\right]
\end{aligned}
$$

In addition, the usual continuity equation is still preserved:

$$
\dot{\rho}_{M G}+3 H\left(\rho_{M G}+p_{M G}\right)=0 .
$$

Variation of the action with respect to the scalar field $\psi$ provides its evolution equation:

$$
\ddot{\psi}+3 H \dot{\psi}+\frac{d W}{d \psi}+\frac{d V}{d \psi}\left\{\left(\frac{a_{r e f}}{a}-1\right)\left[f_{3}(a)+f_{1}(a)\right]+3 \dot{b} f_{2}(a)\right\}=0 .
$$

Additionally, variation with respect to $b$ provides the constraint equation

$$
V(\psi) H f_{1}(a)+\dot{V}(\psi) f_{2}(a)=0,
$$

which, as it was shown in [54], using (2.11) in general leads to

$$
V(\psi(\tau))=V_{0} e^{-\int \frac{f_{1}}{a f_{2}} \mathrm{~d} a}=\frac{V_{0}}{\left(a-a_{r e f}\right)\left[\alpha_{4} a_{r e f}^{2}-\left(3 \alpha_{3}+2 \alpha_{4}\right) a a_{r e f}+\left(3+3 \alpha_{3}+\alpha_{4}\right) a^{2}\right]},
$$

This relation restricts radically the coupling-potential $V(\psi)^{1}$ and can be very helpful since it offers the behavior of $V(\psi(\tau))$ without the need to find explicitly the solution $\psi(\tau)$ [which obviously is consistent with this $V(\psi(\tau))$ ]. Lastly, the equations close by considering the matter evolution equation $\dot{\rho}_{m}+3 H\left(\rho_{m}+p_{m}\right)=0$.

Finally, we mention that from the above expressions we observe that $a_{\text {ref }}$ plays the role of a reference scale factor that can be arbitrary. One could still worry about the fact that $V(\psi)$ in general becomes negative for $a<a_{r e f}$, which can be pushed to very small but still non-zero values, however the bouncing behavior of the present work offers a solution to this problem, since setting the bounce scale factor to be larger than $a_{r e f}$ ensures that the graviton mass square will be always positive. For simplicity throughout this work we set $a_{r e f}=1$.

\section{Bouncing and Cyclic solutions}

The above cosmological scenario proves to exhibit very interesting behavior. In particular, the form of the coupling-potential (2.15) implies that in an expanding universe $V(\psi(\tau))$ always goes to zero at late times, and thus the scenario at hand always gives the standard quintessence scenario [54]. The only case that this will not happen is if the scalar

\footnotetext{
${ }^{1}$ Note that in standard massive gravity this equation imposes strong constraints on $b(\tau)[13,16,51]$, but in the present construction the extra freedom brings these constraints on $V(\psi)$, leaving $b(\tau)$ free [54].
} 
field dynamics is so effective that it will change the universe evolution from expansion to contraction. In this case $V(\psi(\tau))$ will start increasing and at some point it can trigger a nonsingular bounce. A successive sequence of bounces and turnarounds offers the cyclic cosmology. In this section we analytically explore these possibilities.

Whether a universe is expanding or contracting depends on the positivity of the Hubble parameter, that is, in the contracting phase $H$ is negative while in the expanding one it is positive. By making use of the continuity equations it follows that at the bounce and turnaround points $H=0$, however at and around the bounce we have $\dot{H}>0$, while at and around the turnaround we have $\dot{H}<0$. If a bounce and a turnaround can be realized in a given cosmological scenario, then the aforementioned general requirements for $H$ must be fulfilled.

Interestingly enough, observing the form of the two Friedmann equations (2.7), (2.8) along with (2.9), (2.10), (2.15), we can see that the above requirements can be easily fulfilled in the present scenario, for suitable potentials $W(\psi)$ and/or suitable forms for $b(\tau)$. In particular, the coupling-potential $V(\psi)$ can trigger the bounce and the turnaround, and actually it is this term that can cause the necessary null energy condition violation, as it was mentioned in [54], where this term could lead the effective dark energy caused by the gravitational modification to exhibit phantom behavior.

In summary, cosmology governed by extended massive gravity has a large freedom to fulfill the above bounce and cyclicity requirements. In particular, for a given $b(\tau)$ we can adjust the potential $W(\psi)$ in order to obtain a desired scale-factor evolution, or alternatively for a given $W(\psi)$ we can adjust $b(\tau)$. In the following subsections we examine these two cases separately.

\subsection{Known $b(\tau)$}

Suppose we determine $b(\tau)$ at will. Let us first start from the desired result, that is we impose a known form of the scale factor $a(\tau)$ possessing a bouncing or oscillatory behavior. In this case both $H(\tau)$ and $\dot{H}(\tau)$ are straightforwardly known. Therefore, we can use the Friedmann equations (2.7), (2.8) together with $(2.9),(2.10)$, in order to extract the relations for $\psi(\tau)$ (through $\dot{\psi}(\tau)$ ) and $W(\tau)$, obtaining

$$
\begin{gathered}
\begin{aligned}
\psi(\tau)= \pm \int^{\tau} d \tau^{\prime}\left\{-2 M_{P}^{2} \dot{H}\left(\tau^{\prime}\right)\right. & -\rho_{m}\left(a\left(\tau^{\prime}\right)\right)-p_{m}\left(a\left(\tau^{\prime}\right)\right) \\
& \left.+V\left(\psi\left(\tau^{\prime}\right)\right)\left[\dot{b}\left(\tau^{\prime}\right)-\frac{1}{a\left(\tau^{\prime}\right)}\right] f_{1}\left(a\left(\tau^{\prime}\right)\right)\right\}^{\frac{1}{2}}, \\
W(\tau)=M_{P}^{2}\left[3 H^{2}(\tau)+\dot{H}(\tau)\right]+ & \frac{p_{m}(a(\tau))}{2}-\frac{\rho_{m}(a(\tau))}{2} \\
- & V(\psi(\tau))\left\{f_{4}(a(\tau))+\frac{f_{1}(a(\tau))}{2}\left[\dot{b}(\tau)+\frac{1}{a(\tau)}\right]\right\},
\end{aligned}
\end{gathered}
$$

where $\rho_{m}(a(\tau))$ and $p(a(\tau))$ are known as long as the matter equation-of-state parameter $w_{m} \equiv p_{m} / \rho_{m}$ is determined. Then, eliminating time between these two expressions we can extract the explicit form of the potential $W(\psi)$, which is the one that generates the desired $a(\tau)$-form. Finally, we mention that the basic requirement for the above procedure to be 
valid and a bounce or cyclic behavior to be possible, is that the $a(\tau)$ and $b(\tau)$ ansatzes and the parameter choices must lead to a positive $\dot{\psi}^{2}(\tau)$.

We now proceed to a specific, simple, but quite general example. Firstly, we consider $b(\tau)$ to have the simple form

$$
b(\tau)=\tau,
$$

and thus $\dot{b}=1$ in the above expressions (in general we could add a coefficient $b_{0}$ in the above ansatz, but for simplicity we set it equal to 1). Now, in order to get an idea of what kinds of potentials $W(\psi)$ could lead to cyclic behavior, we perform a test-procedure starting from the desired result, that is we assume a cyclic universe with an oscillatory scale factor of the form

$$
a(\tau)=A \sin (\omega \tau)+a_{c},
$$

where the non-zero constant $a_{c}$ is inserted in order to avoid the singularity. Thus, $\tau$ varies between $-\infty$ and $+\infty$, with $\tau=0$ being a specific moment without any particular physical meaning. Finally, note that the bounce occurs at $a_{B}=a_{c}-A$. From (3.4) we straightforwardly find $H(\tau)$ and $\dot{H}(\tau)$, and thus substitution into (2.15) and then to (3.1) and (3.2) gives the corresponding expressions for $\psi(\tau)$ and $W(\tau)$.

In order to provide a more transparent picture of the obtained cosmological behavior, in Fig. 1 we present the evolution of $\psi(\tau)$ and $W(\tau)$ for the scale factor (3.4) with $A=5$, $\omega=0.001$ and $a_{c}=7$, assuming also dust matter $\left(w_{m}=0\right)$, that is $p_{m}=0$ and $\rho_{m}(a)=$ $\rho_{m B} a_{B}^{3} / a^{3}$, with $\rho_{m B}$ the energy density at the bounce. Thus, eliminating time between

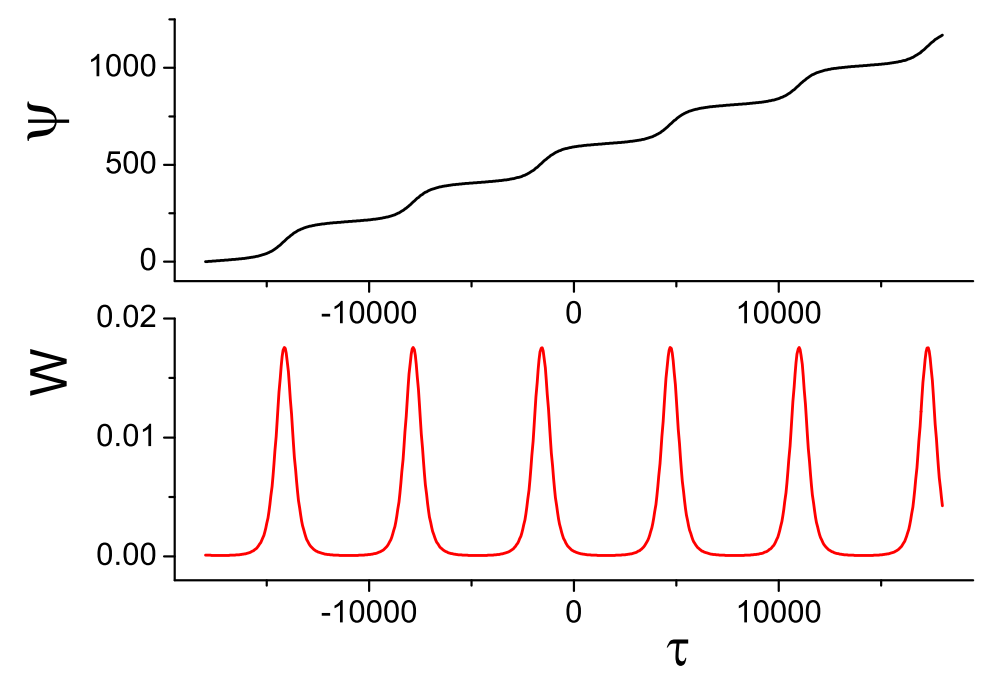

Figure 1. The evolution of $\psi(\tau)$ and $W(\tau)$ as functions of time in the case where $b(\tau)=\tau$ and $a(\tau)=A \sin (\omega \tau)+a_{c}$, with $A=5, \omega=0.001$, and $a_{c}=7$. In numerical elaboration we use $\alpha_{3}=3$, $\alpha_{4}=1, V_{0}=0.2, \rho_{m B}=0.01$, and $M_{P}=1$. All dimensional parameters are normalized in unit of $M_{P}$. 
$\psi(\tau)$ and $W(\tau)$ allows us to re-construct the corresponding expression for $W(\psi)$, shown in Fig. 2. From these figures we observe that an oscillating and non-singular scale factor

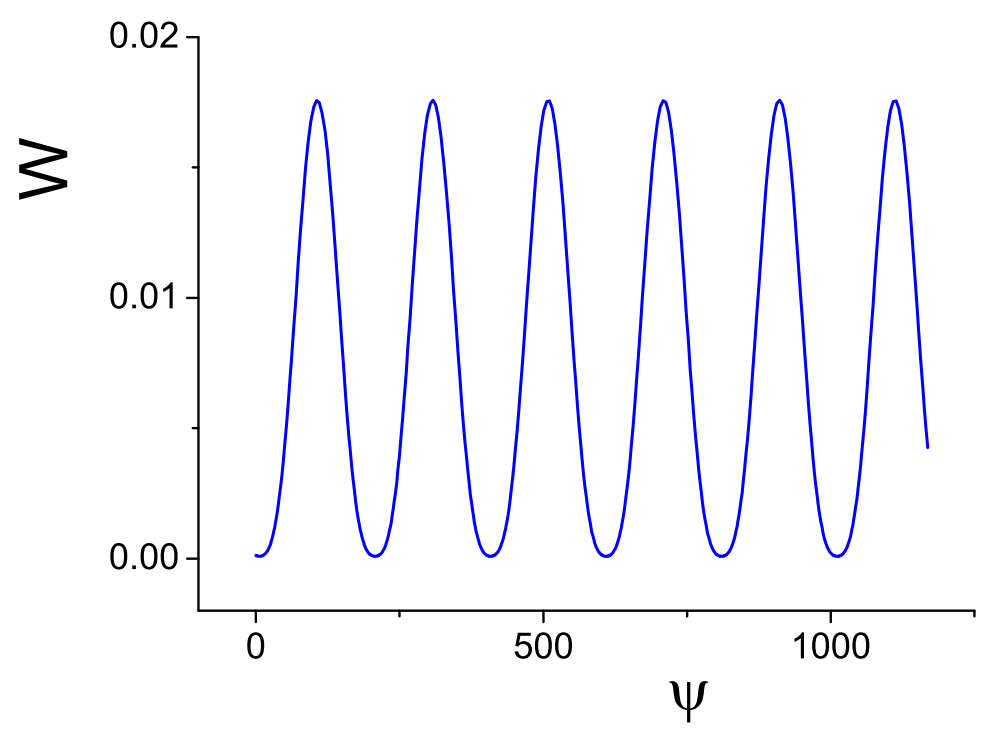

Figure 2. The reconstructed potential $W(\psi)$ in the case where $b(\tau)=\tau$ and $a(\tau)=A \sin (\omega \tau)+a_{c}$, with $A=5, \omega=0.001$, and $a_{c}=7$. In numerical elaboration we use $\alpha_{3}=3, \alpha_{4}=1, V_{0}=0.2$, $\rho_{m B}=0.01$, and $M_{P}=1$. All dimensional parameters are normalized in unit of $M_{P}$.

can be generated by an oscillatory scalar potential $W(\psi)$. This $W(\psi)$-form was expected, since a non-oscillatory $W(\psi)$ would be difficult to produce an infinitely oscillating scale factor. Finally, we mention that although we have presented a specific example, we can straightforwardly repeat the described procedure imposing an arbitrary oscillating form for the scale factor.

The aforementioned bottom-to-top approach was enlightening about the form of the scalar potential that leads to a cyclic cosmological behavior. Therefore, one can perform the above procedure restoring cause and effect, that is starting from a specific oscillatory $W(\psi)$ and resulting to an oscillatory $a(\tau)$. In particular, $(3.1)$ is written in a compact form as $\dot{\psi}^{2}(\tau)=P_{1}(a, \dot{a}, \ddot{a})$ and similarly $(3.2)$ as $W(\tau)=P_{2}(a, \dot{a}, \ddot{a})$. Thus, we can invert the known form of $W(\psi) \equiv W(\psi(\tau))$ acquiring $\psi(\tau)=W^{\{-1\}}\left(P_{2}(a, \dot{a}, \ddot{a})\right)$, and then $\dot{\psi}^{2}(\tau)=\left\{\frac{d}{d \tau}\left[W^{\{-1\}}\left(P_{2}(a, \dot{a}, \ddot{a})\right)\right]\right\}^{2}$. Therefore, the scale factor arises as a solution of the differential equation

$$
P_{1}(a, \dot{a}, \ddot{a})=\left\{\frac{d}{d \tau}\left[W^{\{-1\}}\left(P_{2}(a, \dot{a}, \ddot{a})\right)\right]\right\}^{2}
$$

As a specific example we consider the simple form

$$
W(\psi)=W_{0} \sin \left(\omega_{W} \psi\right)+W_{c}
$$


In this case $\psi=\frac{1}{\omega_{W}} \sin ^{-1}\left[\frac{W(\psi(\tau))-W_{c}}{W_{0}}\right]$, where $W(\psi(\tau)) \equiv W(\tau)=P_{2}(a, \dot{a}, \ddot{a})$ with $P_{2}(a, \dot{a}, \ddot{a})$ the right hand side of expression (3.2). Therefore, the differentiation leads to:

$$
\dot{\psi}(\tau)=\frac{1}{W_{0} \omega_{W}} \frac{1}{\sqrt{1-\left[\frac{P_{2}(a, \dot{a}, \ddot{a})-W_{c}}{W_{0}}\right]^{2}}} \frac{d}{d \tau}\left[P_{2}(a, \dot{a}, \ddot{a})\right]
$$

and thus we obtain

$$
P_{1}(a, \dot{a}, \ddot{a})=\left\{\frac{1}{W_{0} \omega_{W}} \frac{1}{\sqrt{1-\left[\frac{P_{2}(a, \dot{a}, \ddot{a})-W_{c}}{W_{0}}\right]^{2}}} \frac{d}{d \tau}\left[P_{2}(a, \dot{a}, \ddot{a})\right]\right\}^{2},
$$

where as we have mentioned $P_{1}(a, \dot{a}, \ddot{a})$ is the expression inside the curly brackets in (3.1).

Differential equation (3.8) cannot be solved analytically, but it can be easily elaborated numerically, and moreover inserting its solution for $a(\tau)$ into $(3.7)$ we obtain $\psi(\tau)$ too. In Fig. 3 we depict the corresponding solutions for $a(\tau)$ and $\psi(\tau)$, for the ansatz (3.6) with $W_{0}=0.01, \omega_{W}=0.025$ and $W_{c}=0.01$, for $\alpha_{3}=3, \alpha_{4}=1, V_{0}=0.2, \rho_{m B}=0.01, M_{P}=1$, in $M_{P}^{2}$-units (the potential parameters have been chosen in order to acquire a cyclic universe with $a_{B} \approx 2$ at the bounce, similarly to the previous example). In conclusion, we verified

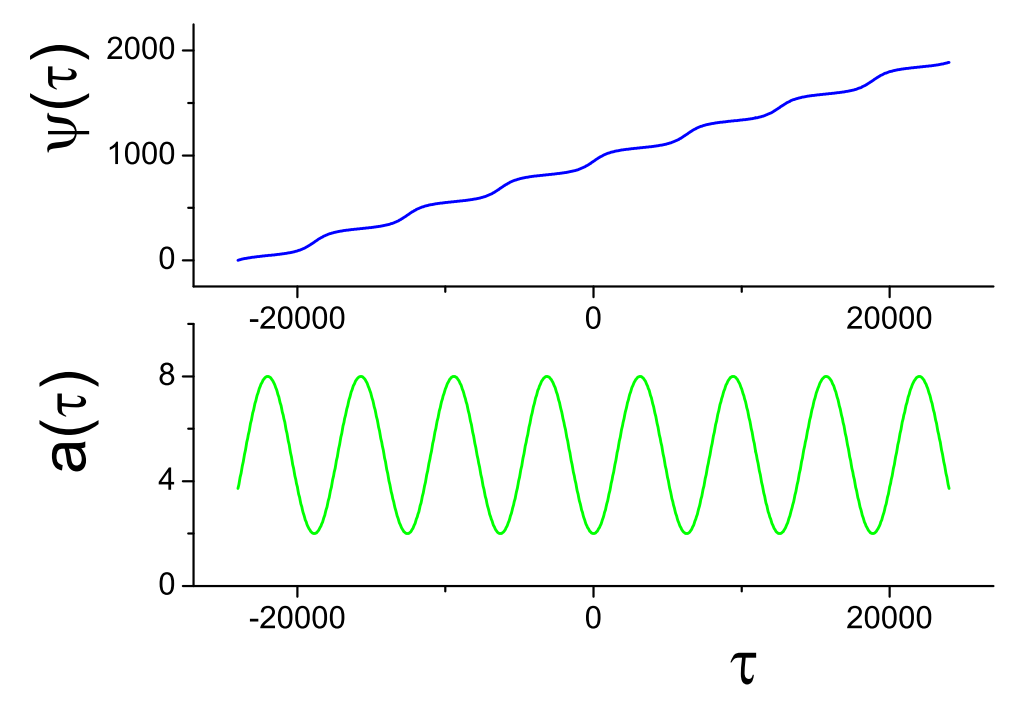

Figure 3. The evolution of the scalar field $\psi(\tau)$ (upper graph) and of the scale factor (lower graph), for $b(\tau)=\tau$ and for the scalar-field potential $W(\psi)=W_{0} \sin \left(\omega_{W} \psi\right)+W_{c}$ with $W_{0}=0.01$, $\omega_{W}=0.025$ and $W_{c}=0.01$. In numerical elaboration we use $\alpha_{3}=3, \alpha_{4}=1, V_{0}=0.2, \rho_{m B}=0.01$, and $M_{P}=1$. All dimensional parameters are normalized in unit of $M_{P}$.

that an oscillatory scalar-field potential can lead to a cyclic universe. 
We close this subsection by mentioning that here $b(\tau)$ was considered by hand and then we determined the potential $W(\psi)$ in order to obtain cyclic behavior. The only requirement for the above analysis to be valid is to obtain a non-negative $\dot{\psi}^{2}$ in (3.1). If this is not possible we deduce that the corresponding parameter-choice and/or the imposed $b(\tau)$-ansatz cannot lead to a cyclic solution. However, note that there are $b(\tau)$ ansatzes that cannot lead to cyclicity independently of the parameter choice (for $\alpha_{3}, \alpha_{4}, V_{0}, \rho_{m B}, w_{m}$ ). In particular, using the forms $b(\tau)=a(\tau)$ and $b(\tau)=\ln a(\tau)$ (which in [54] it was shown to lead to phantom behavior) we could not find any parameter choice that could lead to non-negative $\dot{\psi}^{2}$ and therefore to a cyclic behavior. Thus, cyclicity cannot be obtained for every $b(\tau)$-form.

\section{$3.2 \quad$ Known $W(\psi)$}

Suppose we determine $W(\psi)$ at will, and similarly to the previous subsection as a first test-procedure we impose a known scale factor $a(\tau)$ possessing oscillatory behavior, that is both $H(\tau)$ and $\dot{H}(\tau)$ are known. In this case eliminating $\dot{\psi}$ between the two Friedmann equations $(2.7),(2.8)$ (with $a_{r e f}=1$ ) straightforwardly gives

$$
\begin{aligned}
\dot{b}(\tau)=\frac{1}{a(\tau)} & +\left[V(a(\tau)) f_{1}(\tau)\right]^{-1}\left\{2 M_{P}^{2}\left[\dot{H}(\tau)+3 H^{2}(\tau)\right]-\rho_{m}(a(\tau))+p_{m}(a(\tau))\right. \\
& \left.-2 W(\psi(\tau))-2 V(a(\tau))\left[\frac{1}{a(\tau)}-1\right]\left[f_{3}(a(\tau))+f_{1}(a(\tau))\right]\right\} \equiv P_{3}(\psi, \tau),
\end{aligned}
$$

where $V(a(\tau))$ is given by (2.15), and thus substitution to the scalar-field evolution equation (2.13), along with the chain rule $\frac{d V}{d \psi}=\frac{d V}{d a} \frac{\dot{a}}{\dot{\psi}}$, leads to a differential equation for $\psi$ of the form

$\ddot{\psi}+3 H \dot{\psi}+\frac{d W}{d \psi}+\frac{d V}{d a} \frac{\dot{a}}{\dot{\psi}}\left\{\left(\frac{1}{a}-1\right)\left[f_{3}(a)+f_{1}(a)\right]+3 P_{3}(\psi, \tau) f_{2}(a)\right\} \equiv P_{4}(\psi, \dot{\psi}, \ddot{\psi}, t)=0$,

that can be numerically solved, leading to $\psi(\tau)$. Thus, inserting $\psi(\tau)$ back to (3.9) gives a simple differential equation of the form $\dot{b}=P_{5}(\tau)$ that can be easily solved as

$$
b(\tau)=\int^{\tau} P_{5}\left(\tau^{\prime}\right) d \tau^{\prime} .
$$

As a specific example we consider the simplest case in which $W(\psi)$ is constant, namely

$$
W(\psi)=W_{0}
$$

Similarly to the previous subsection we assume a cyclic universe with the oscillatory scale factor (3.4), namely $a(\tau)=A \sin (\omega \tau)+a_{c}$. In this case from (3.9) we can see that $\dot{b}(\tau)$ is analytically known, which simplifies the procedure. Assuming also dust matter $\left(w_{m}=0\right)$ and $\rho_{m}(a)=\rho_{m B} a_{B}^{3} / a^{3}$, with $\rho_{m B}$ the energy density at the bounce, we numerically solve the differential equation (3.10), in order to extract $\psi(\tau)$. In Fig. 4 we depict the corresponding evolution of $\psi(\tau), \dot{b}(\tau)$ and $b(\tau)$, and thus we deduce that this $b(\tau)$ form generates the oscillatory universe. As we observe, $\dot{b}(\tau)$ has an oscillatory profile, although 


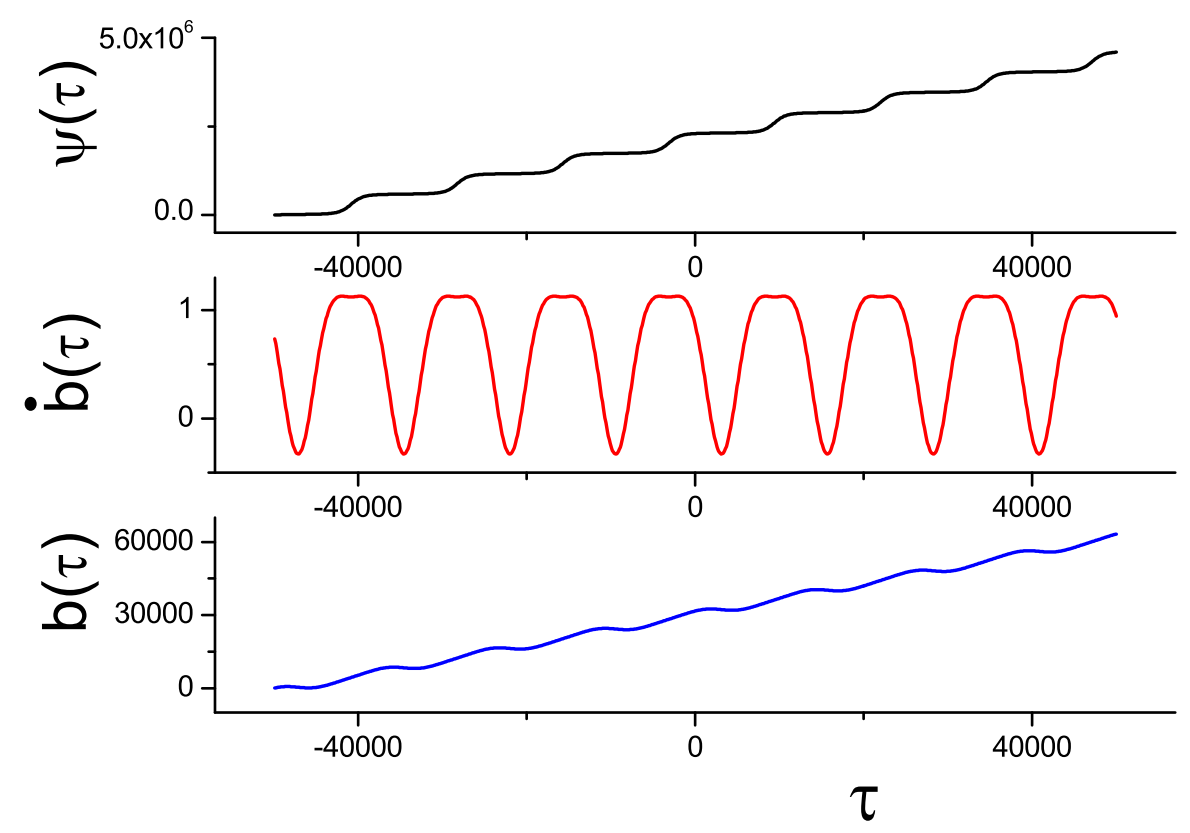

Figure 4. The evolution of the scalar field $\psi(\tau)$ (upper graph), of the time-derivative of the Stückelberg-scalar function $\dot{b}(\tau)$ (middle graph) and of the Stückelberg-scalar function b $\tau$ ) (lower graph), in the case where $W(\psi)=W_{0}$ with $W_{0}=0.001$ and $a(\tau)=A \sin (\omega \tau)+a_{c}$, with $A=3$, $\omega=0.0005$, and $a_{c}=5$. In numerical elaboration we use $\alpha_{3}=10, \alpha_{4}=10, V_{0}=0.6, \rho_{m B}=0.1$, and $M_{P}=10$. All dimensional parameters are normalized in unit of $M_{P}$.

not of a simple form. The fact that $\dot{b}(\tau)$ and not $b(\tau)$ should be oscillatory in order to produce an oscillatory scale factor was expected, since in the cosmological equations it is $\dot{b}$ and not $b$ that appears.

We stress here that the differential equation (3.10) in the above procedure cannot be solved for every $W(\psi)$, and additionally one has to suitably choose the model parameters. Thus, although in the previous subsection one had full control on which cases lead to oscillatory solutions (namely those that give a positive $\dot{\psi}^{2}(\tau)$ in $(3.1)$ ), in the present subsection one cannot easily see which $W(\psi)$ ansatzes and/or which parameter choices can lead to cyclic solutions.

Finally, since from the above bottom-to-top approach we deduced that in order to get an oscillatory universe we must use an oscillatory $\dot{b}(\tau)$, in the following we restore cause and effect and we impose such a $\dot{b}(\tau)$ as an input, extracting the resulting $\psi(\tau)$ and $a(\tau)$ from the solutions of the cosmological equations. Although this procedure is possible in general, in the following we apply it in the simple example of a constant $W(\psi)=W_{0}$ of (3.12). In this case, and with a given $\dot{b}(\tau)$, equation (3.9) is a simple differential equation for $a(\tau)$, which can be numerically solved. Then, inserting this $a(\tau)$ back in (3.1), we find 
also the solution for $\psi(\tau)$. Similarly to the previous subsection, the only requirement for this procedure to work is to obtain a positive $\dot{\psi}^{2}(\tau)$.

As a specific example for $\dot{b}(\tau)$ we impose the simple ansatz

$$
\dot{b}(\tau)=B \sin \left(\omega_{B} \tau\right)+b_{c},
$$

that is we impose a Stückelberg-scalar function of the form

$$
b(\tau)=-\frac{B}{\omega_{B}} \cos \left(\omega_{B} \tau\right)+b_{c} \tau+c,
$$

with $c$ an integration constant. We then solve numerically (3.9) for $a(\tau)$ and (3.1) for $\psi(\tau)$ and in Fig. 5 we depict the corresponding results. Indeed, the Stückelberg-scalar function
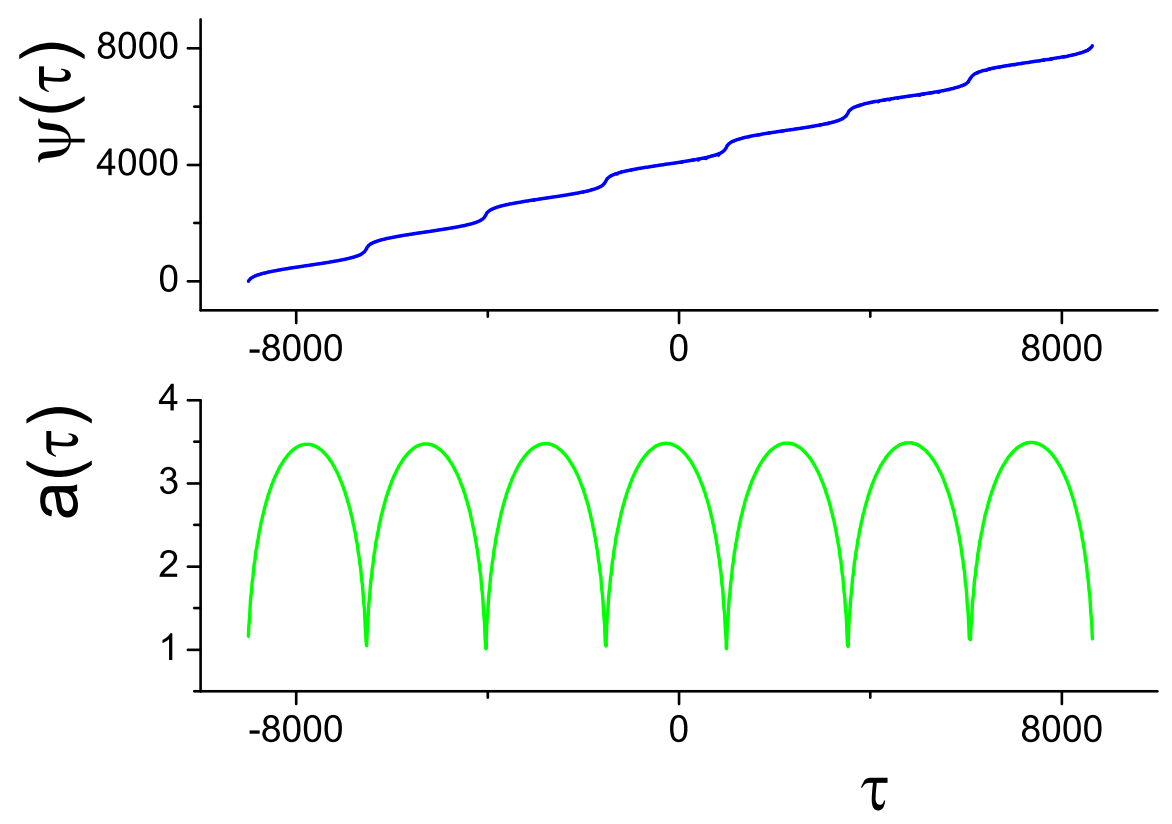

Figure 5. The evolution of the scalar field $\psi(\tau)$ (upper graph) and of the scale factor (lower graph), in the case where $W(\psi)=W_{0}$ and $\dot{b}(\tau)=B \sin \left(\omega_{B} \tau\right)+b_{c}$, with $W_{0}=0.001, B=1, \omega_{b}=10^{-6}$ and $b_{c}=2$. In numerical elaboration we use $\alpha_{3}=4, \alpha_{4}=4, V_{0}=1, \rho_{m B}=0.0005$, and $M_{P}=100$. All dimensional parameters are normalized in unit of $M_{P}$.

(3.14) generates an oscillatory universe, although not of a simple form since the turnarounds are softer than the bounces. However, we mention that one needs to carefully select the model parameters in order to obtain an oscillatory scale factor under the $b(\tau)$-ansatz (3.14), however he has control of the procedure, since he needs to fulfill the requirement of $\dot{\psi}^{2}(\tau)$ to be positive. 


\section{Discussion}

In the present work we investigated bouncing and cyclic cosmological behaviors in a universe governed by extended massive gravity, in which the graviton mass has been promoted to a function of an extra scalar field. This model has additional freedom comparing to usual massive gravity, and thus it leads to significantly different and richer behavior [53, 54]. In particular, although the scenario at late times tends to coincide with standard quintessence, at early and intermediate times the effective graviton mass can be large and thus play a crucial role in the universe evolution. Amongst others, the capability of the scenario to lead to violation of the Null Energy Condition can lead to a bounce or a turnaround, the successive sequence of which can naturally give rise to cyclic cosmology.

Extended nonlinear massive gravity has an enhanced freedom (apart from the extra scalar field one can see that the Stückelberg-scalar function $b(\tau)$ is not constrained as in usual massive gravity and it can be free), therefore it can drive cyclicity with two different mechanisms. The first is to impose an arbitrary Stückelberg-scalar function $b(\tau)$ and suitably choose the usual scalar field potential $W(\psi)$ in order to obtain a cyclic behavior. It proves that one should use an oscillating $W(\psi)$ as expected, and the only requirement for this procedure to hold is to obtain a positive $\dot{\psi}^{2}(\tau)$. Thus, this is not possible for every $b(\tau)$ form, that is not every $b(\tau)$ can be consistent with cyclicity.

The second mechanism to drive cyclic behavior is exactly the Stückelberg-scalar function $b(\tau)$. In particular, imposing an arbitrary scalar-field potential $W(\psi)$ we suitably choose $b(\tau)$ in order to obtain a cyclic behavior. It proves that one should use an oscillating $\dot{b}(\tau)$ as expected (since $\dot{b}(\tau)$ appears in the equations and not $b(\tau)$ ). Similarly to the first mechanism above, not all scalar potentials are consistent with cyclicity.

A crucial issue in all bouncing and cyclic scenarios is the processing of perturbations through the bounces. A simple check on the stability of this type of models is to look at the generalized Higuchi bound derived in [48] (see also [118] for a different expression in an earlier model). In particular, as an effective theory, nonlinear massive gravity is reliable only when the scale is well beneath the cut off $\Lambda_{3}=\left(M_{P} m_{g}^{2}\right)^{1 / 3}$, where $m_{g}^{2}$ is the graviton mass square, since upon $\Lambda_{3}$ helicity 0 mode couples strongly to helicity 1 and helicity 2 modes, and thus effective field theory breaks down. Therefore, in the present extended scenario one should compare ${ }^{2}$ the various appeared scales, such are $H, \dot{H}, \ddot{H}$ etc, with $\Lambda_{3}=\left[M_{P} V(\psi)\right]^{1 / 3}$. Indeed, in all the above examples $H / \Lambda_{3} \lesssim 10^{-3}$, while $\dot{H} / \Lambda_{3} \lesssim 10^{-5}, \ddot{H} / \Lambda_{3} \lesssim 10^{-7}$ etc, and thus the scenario is reliable. On the other hand note that $V(\psi)$ is always much smaller than $M_{P}^{2}\left(V(\psi) / M_{P}^{2} \lesssim 10^{-3}\right.$ and $\left.V_{0} / M_{P}^{2} \lesssim 10^{-1}\right)$, which is an additional requirement for the robustness of the scenario. Therefore, at this level, we can conclude that our model is well behaved when the perturbations pass through the bouncing points. However, we should notice that since a cosmic scalar is introduced to drive the graviton mass varying along background evolution, the stability issue arisen from this scalar field ought to be taken into account in a global analysis. Such a complete perturbation analysis of the extended nonlinear massive gravity lies beyond the scope of the present work and it is left for future investigation.

\footnotetext{
${ }^{2}$ We wish to thank the referee for mentioning this point.
} 


\section{Acknowledgments}

We wish to thank S. Dutta, Q-G. Huang, Y.-S, Piao, S.-Y. Zhou and an anonymous referee, for useful comments. The work of CYF is supported in part by the Cosmology Initiative in Arizona State University. The work of GCX is supported in part by the Summer Research Assistantship from the Graduate School of the University of Mississippi and in part by the Cosmology Initiative in Arizona State University. The research of ENS is implemented within the framework of the Action Supporting Postdoctoral Researchers of the Operational Program "Education and Lifelong Learning" (Actions Beneficiary: General Secretariat for Research and Technology), and is co-financed by the European Social Fund (ESF) and the Greek State.

\section{References}

[1] M. Fierz, W. Pauli, On relativistic wave equations for particles of arbitrary spin in an electromagnetic field, Proc. Roy. Soc. Lond. A173, 211 (1939).

[2] A. I. Vainshtein, To the problem of nonvanishing gravitation mass, Phys. Lett. B 39, 393 (1972).

[3] H. van Dam, M. J. G. Veltman, Massive and massless Yang-Mills and gravitational fields, Nucl. Phys. B22, 397 (1970).

[4] V. I. Zakharov, Linearized gravitation theory and the graviton mass, JETP Lett. 12, 312 (1970).

[5] D. G. Boulware, S. Deser, Can gravitation have a finite range?, Phys. Rev. D6, 3368 (1972).

[6] C. de Rham, G. Gabadadze, Generalization of the Fierz-Pauli Action, Phys. Rev. D82, 044020 (2010), [arXiv: 1007.0443].

[7] C. de Rham, G. Gabadadze and A. J. Tolley, Resummation of Massive Gravity, Phys. Rev. Lett. 106, 231101 (2011), [arXiv: 1011.1232].

[8] K. Hinterbichler, Theoretical Aspects of Massive Gravity, Rev. Mod. Phys. 84, 671 (2012), [arXiv: 1105.3735].

[9] K. Koyama, G. Niz and G. Tasinato, Strong interactions and exact solutions in non-linear massive gravity, Phys. Rev. D 84 (2011) 064033, [arXiv:1104.2143].

[10] S. F. Hassan and R. A. Rosen, Resolving the Ghost Problem in non-Linear Massive Gravity, Phys. Rev. Lett. 108, 041101 (2012), [arXiv: 1106.3344].

[11] C. de Rham, G. Gabadadze and A. Tolley, Ghost free Massive Gravity in the Stúckelberg language, Phys. Lett. B 711, 190 (2012), [arXiv:1107.3820].

[12] B. Cuadros-Melgar, E. Papantonopoulos, M. Tsoukalas and V. Zamarias, Massive Gravity with Anisotropic Scaling, [arXiv:1108.3771].

[13] G. D'Amico, C. de Rham, S. Dubovsky, G. Gabadadze, D. Pirtskhalava, A. J. Tolley, Massive Cosmologies, [arXiv:1108.5231].

[14] S. F. Hassan and R. A. Rosen, Bimetric Gravity from Ghost-free Massive Gravity, JHEP 1202, 126 (2012), [arXiv:1109.3515]. 
[15] J. Kluson, Note About Hamiltonian Structure of Non-Linear Massive Gravity, JHEP 1201, 013 (2012), [arXiv: 1109.3052].

[16] A. E. Gumrukcuoglu, C. Lin and S. Mukohyama, Open FRW universes and self-acceleration from nonlinear massive gravity, JCAP 1111, 030 (2011), [arXiv:1109.3845].

[17] M. S. Volkov, Cosmological solutions with massive gravitons in the bigravity theory, JHEP 1201, 035 (2012), [arXiv: 1110.6153].

[18] M. von Strauss, A. Schmidt-May, J. Enander, E. Mortsell and S. F. Hassan, Cosmological Solutions in Bimetric Gravity and their Observational Tests, JCAP 1203, 042 (2012), [arXiv: 1111.1655].

[19] D. Comelli, M. Crisostomi, F. Nesti and L. Pilo, FRW Cosmology in Ghost Free Massive Gravity, JHEP 1203, 067 (2012) [Erratum-ibid. 1206, 020 (2012)], [arXiv:1111.1983].

[20] S. F. Hassan and R. A. Rosen, Confirmation of the Secondary Constraint and Absence of Ghost in Massive Gravity and Bimetric Gravity, JHEP 1204, 123 (2012), [arXiv:1111.2070].

[21] L. Berezhiani, G. Chkareuli, C. de Rham, G. Gabadadze and A. J. Tolley, On Black Holes in Massive Gravity, Phys. Rev. D 85, 044024 (2012), [arXiv:1111.3613].

[22] A. E. Gumrukcuoglu, C. Lin and S. Mukohyama, Cosmological perturbations of self-accelerating universe in nonlinear massive gravity, JCAP 1203, 006 (2012), [arXiv: 1111.4107].

[23] N. Khosravi, N. Rahmanpour, H. R. Sepangi and S. Shahidi, Multi-Metric Gravity via Massive Gravity, Phys. Rev. D 85, 024049 (2012), [arXiv:1111.5346].

[24] Y. Brihaye and Y. Verbin, Perfect Fluid Spherically-Symmetric Solutions in Massive Gravity, [arXiv:1112.1901].

[25] M. Park, Quantum Aspects of Massive Gravity, Class. Quant. Grav. 28, 105012 (2011), [arXiv: 1009.4369].

[26] M. Park, Quantum Aspects of Massive Gravity II: Non-Pauli-Fierz Theory, JHEP 1110, 130 (2011), [arXiv: 1011.4266].

[27] I. L. Buchbinder, D. D. Pereira and I. L. Shapiro, One-loop divergences in massive gravity theory, Phys. Lett. B 712, 104 (2012), [arXiv:1201.3145].

[28] H. Ahmedov and A. N. Aliev, Type N Spacetimes as Solutions of Extended New Massive Gravity, Phys. Lett. B 711, 117 (2012), [arXiv:1201.5724].

[29] E. A. Bergshoeff, J. J. Fernandez-Melgarejo, J. Rosseel and P. K. Townsend, On 'New Massive’ 4D Gravity, JHEP 1204, 070 (2012), [arXiv:1202.1501].

[30] M. Crisostomi, D. Comelli and L. Pilo, Perturbations in Massive Gravity Cosmology, [arXiv: 1202.1986].

[31] M. F. Paulos and A. J. Tolley, Massive Gravity Theories and limits of Ghost-free Bigravity models, [arXiv: 1203.4268].

[32] S. F. Hassan, A. Schmidt-May and M. von Strauss, Proof of Consistency of Nonlinear Massive Gravity in the Stúckelberg Formulation, [arXiv:1203.5283].

[33] D. Comelli, M. Crisostomi, F. Nesti and L. Pilo, Degrees of Freedom in Massive Gravity, [arXiv: 1204.1027]. 
[34] F. Sbisa, G. Niz, K. Koyama and G. Tasinato, Characterising Vainshtein Solutions in Massive Gravity, [arXiv:1204.1193].

[35] J. Kluson, Non-Linear Massive Gravity with Additional Primary Constraint and Absence of Ghosts, [arXiv: 1204.2957].

[36] G. Tasinato, K. Koyama and G. Niz, New symmetries in Fierz-Pauli massive gravity, [arXiv: 1204.5880].

[37] K. Morand and S. N. Solodukhin, Dual Massive Gravity, [arXiv:1204.6224].

[38] V. F. Cardone, N. Radicella and L. Parisi, Constraining massive gravity with recent cosmological data, Phys. Rev. D 85, 124005 (2012), [arXiv:1205.1613].

[39] V. Baccetti, P. Martin-Moruno and M. Visser, Massive gravity from bimetric gravity, [arXiv: 1205.2158].

[40] P. Gratia, W. Hu and M. Wyman, Self-accelerating Massive Gravity: Exact solutions for any isotropic matter distribution, [arXiv:1205.4241].

[41] M. S. Volkov, Exact self-accelerating cosmologies in the ghost-free bigravity and massive gravity, [arXiv:1205.5713].

[42] A. De Felice, A. E. Gumrukcuoglu and S. Mukohyama, Massive gravity: nonlinear instability of the homogeneous and isotropic universe, [arXiv:1206.2080].

[43] A. E. Gumrukcuoglu, C. Lin and S. Mukohyama, Anisotropic Friedmann-Robertson-Walker universe from nonlinear massive gravity, [arXiv:1206.2723].

[44] C. de Rham and S. Renaux-Petel, Massive Gravity on de Sitter and Unique Candidate for Partially Massless Gravity, [arXiv:1206.3482].

[45] M. Berg, I. Buchberger, J. Enander, E. Mortsell and S. Sjors, Growth Histories in Bimetric Massive Gravity, [arXiv:1206.3496].

[46] G. D'Amico, Cosmology and perturbations in massive gravity, [arXiv:1206.3617].

[47] V. Baccetti, P. Martin-Moruno and M. Visser, Null Energy Condition violations in bimetric gravity, [arXiv:1206.3814].

[48] M. Fasiello and A. J. Tolley, Cosmological perturbations in Massive Gravity and the Higuchi bound, [arXiv: 1206.3852].

[49] G. D'Amico, G. Gabadadze, L. Hui and D. Pirtskhalava, Quasi-Dilaton: Theory and Cosmology, [arXiv: 1206.4253].

[50] V. Baccetti, P. Martin-Moruno and M. Visser, Gordon and Kerr-Schild ansatze in massive and bimetric gravity, [arXiv:1206.4720].

[51] D. Langlois and A. Naruko, Cosmological solutions of massive gravity on de Sitter, [arXiv:1206.6810].

[52] Y. Gong, Cosmology in massive gravity, [arXiv:1207.2726].

[53] Q. -G. Huang, Y. -S. Piao and S. -Y. Zhou, Mass-Varying Massive Gravity, [arXiv: 1206.5678].

[54] E. N. Saridakis, Phantom crossing and quintessence limit in extended nonlinear massive gravity, [arXiv:1207.1800].

[55] A. Borde and A. Vilenkin, Eternal Inflation And The Initial Singularity, Phys. Rev. Lett. 
72, 3305 (1994), [arXiv: gr-qc/9312022].

[56] V. F. Mukhanov and R. H. Brandenberger, A Nonsingular universe, Phys. Rev. Lett. 68, 1969 (1992).

[57] R. H. Brandenberger, V. F. Mukhanov and A. Sornborger, A Cosmological theory without singularities, Phys. Rev. D 48, 1629 (1993), [arXiv:gr-qc/9303001].

[58] Y. -F. Cai, D. A. Easson and R. Brandenberger, Towards a Nonsingular Bouncing Cosmology, [arXiv: 1206.2382].

[59] G. Veneziano, Scale Factor Duality For Classical And Quantum Strings, Phys. Lett. B 265, 287 (1991).

[60] J. Khoury, B. A. Ovrut, P. J. Steinhardt and N. Turok, The ekpyrotic universe: Colliding branes and the origin of the hot big bang, Phys. Rev. D 64, 123522 (2001), [arXiv: hep-th/0103239].

[61] R. Brustein and R. Madden, A model of graceful exit in string cosmology, Phys. Rev. D 57, 712 (1998), [arXiv:hep-th/9708046].

[62] A. Kehagias and E. Kiritsis, Mirage cosmology, JHEP 9911, 022 (1999), [arXiv:hep-th/9910174].

[63] Y. Shtanov and V. Sahni, Bouncing braneworlds, Phys. Lett. B 557, 1 (2003), [arXiv: gr-qc/0208047].

[64] E. N. Saridakis, Cyclic Universes from General Collisionless Braneworld Models, Nucl. Phys. B 808, 224 (2009), [arXiv:0710.5269].

[65] Y. -F. Cai and E. N. Saridakis, Cyclic cosmology from Lagrange-multiplier modified gravity, Class. Quant. Grav. 28, 035010 (2011), [arXiv: 1007.3204].

[66] Y. -F. Cai, S. -H. Chen, J. B. Dent, S. Dutta and E. N. Saridakis, Matter Bounce Cosmology with the $f(T)$ Gravity, Class. Quant. Grav. 28, 215011 (2011), [arXiv: 1104.4349].

[67] R. Brandenberger, Matter Bounce in Horava-Lifshitz Cosmology, Phys. Rev. D 80, 043516 (2009), [arXiv:0904.2835].

[68] Y. F. Cai and E. N. Saridakis, Non-singular cosmology in a model of non-relativistic gravity, JCAP 0910, 020 (2009), [arXiv: 0906.1789].

[69] E. N. Saridakis, Horava-Lifshitz Dark Energy, Eur. Phys. J. C 67, 229 (2010), [arXiv: 0905.3532].

[70] G. Leon and E. N. Saridakis, Phase-space analysis of Horava-Lifshitz cosmology, JCAP 0911, 006 (2009), [arXiv:0909.3571].

[71] M. Bojowald, Absence of singularity in loop quantum cosmology, Phys. Rev. Lett. 86, 5227 (2001), [arXiv: gr-qc/0102069].

[72] J. Martin and P. Peter, Parametric amplification of metric fluctuations through a bouncing phase, Phys. Rev. D 68, 103517 (2003), [arXiv:hep-th/0307077].

[73] E. N. Saridakis and S. V. Sushkov, Quintessence and phantom cosmology with non-minimal derivative coupling, Phys. Rev. D 81, 083510 (2010). [arXiv: 1002.3478].

[74] T. Biswas, T. Koivisto and A. Mazumdar, Towards a resolution of the cosmological singularity in non-local higher derivative theories of gravity, JCAP 1011, 008 (2010), [arXiv: 1005.0590]. 
[75] G. Leon and E. N. Saridakis, Dynamics of the anisotropic Kantowsky-Sachs geometries in $R^{n}$ gravity, Class. Quant. Grav. 28, 065008 (2011), [arXiv: 1007.3956].

[76] T. Biswas, E. Gerwick, T. Koivisto and A. Mazumdar, Towards singularity and ghost free theories of gravity, Phys. Rev. Lett. 108, 031101 (2012), [arXiv:1110.5249].

[77] T. Biswas, A. S. Koshelev, A. Mazumdar and S. Y. Vernov, Stable bounce and inflation in non-local higher derivative cosmology, [arXiv:1206.6374].

[78] M. P. Dabrowski, T. Stachowiak and M. Szydlowski, Phantom cosmologies, Phys. Rev. D 68, 103519 (2003), [arXiv:hep-th/0307128].

[79] M. P. Dabrowski and T. Stachowiak, Phantom Friedmann cosmologies and higher-order characteristics of expansion, Annals Phys. 321, 771 (2006), [arXiv:hep-th/0411199].

[80] Y. F. Cai, T. Qiu, Y. S. Piao, M. Li and X. Zhang, Bouncing Universe with Quintom Matter, JHEP 0710, 071 (2007), [arXiv:0704.1090].

[81] Y. F. Cai, T. T. Qiu, J. Q. Xia and X. Zhang, A Model Of Inflationary Cosmology Without Singularity, Phys. Rev. D 79, 021303 (2009), [arXiv:0808.0819].

[82] Y. F. Cai, T. Qiu, R. Brandenberger, Y. S. Piao and X. Zhang, On Perturbations of Quintom Bounce, JCAP 0803, 013 (2008), [arXiv:0711.2187].

[83] Y. F. Cai and X. Zhang, Evolution of Metric Perturbations in Quintom Bounce model, JCAP 0906, 003 (2009), [arXiv:0808.2551].

[84] Y. -F. Cai, T. -t. Qiu, R. Brandenberger and X. -m. Zhang, A Nonsingular Cosmology with a Scale-Invariant Spectrum of Cosmological Perturbations from Lee-Wick Theory, Phys. Rev. D 80, 023511 (2009), [arXiv:0810.4677].

[85] P. Creminelli and L. Senatore, A Smooth bouncing cosmology with scale invariant spectrum, JCAP 0711, 010 (2007), [arXiv: hep-th/0702165].

[86] E. I. Buchbinder, J. Khoury and B. A. Ovrut, New Ekpyrotic cosmology, Phys. Rev. D 76, 123503 (2007), [arXiv:hep-th/0702154].

[87] C. Lin, R. H. Brandenberger and L. Levasseur Perreault, A Matter Bounce By Means of Ghost Condensation, JCAP 1104, 019 (2011), [arXiv: 1007.2654].

[88] T. Biswas, A. Mazumdar and W. Siegel, Bouncing universes in string-inspired gravity, JCAP 0603, 009 (2006), [arXiv:hep-th/0508194].

[89] S. Alexander, T. Biswas and R. H. Brandenberger, On the Transfer of Adiabatic Fluctuations through a Nonsingular Cosmological Bounce, [arXiv:0707.4679].

[90] T. Qiu, J. Evslin, Y. -F. Cai, M. Li and X. Zhang, Bouncing Galileon Cosmologies, JCAP 1110, 036 (2011), [arXiv:1108.0593].

[91] D. A. Easson, I. Sawicki and A. Vikman, G-Bounce, JCAP 1111, 021 (2011), [arXiv: 1109.1047].

[92] L. R. Abramo and P. Peter, K-Bounce, JCAP 0709, 001 (2007), [arXiv:0705.2893].

[93] A.A Starobinskii, On a nonsingular isotropic cosmological model, Soviet. Astronomy. Letters. vol 4, 82 (1978), [www.adsabs.harvard.edu/full/1978SvAL....4...82S].

[94] D. N. Page, A Fractal Set of Perpetually Bouncing Universes?, Class. Quant. Grav. 1, 417 (1984).

[95] Y. -F. Cai and X. Zhang, Primordial perturbation with a modified dispersion relation, Phys. 
Rev. D 80, 043520 (2009), [arXiv:0906.3341].

[96] Y. -F. Cai, W. Xue, R. Brandenberger and X. -m. Zhang, Thermal Fluctuations and Bouncing Cosmologies, JCAP 0906, 037 (2009), [arXiv:0903.4938].

[97] Y. -F. Cai, W. Xue, R. Brandenberger and X. Zhang, Non-Gaussianity in a Matter Bounce, JCAP 0905, 011 (2009), [arXiv: 0903.0631].

[98] J. Liu, Y. -F. Cai and H. Li, Evidences for bouncing evolution before inflation in cosmological surveys, J. Theor. Phys. 1, 1 (2012) [arXiv:1009.3372].

[99] Y. -F. Cai, R. Brandenberger and X. Zhang, The Matter Bounce Curvaton Scenario, JCAP 1103, 003 (2011), [arXiv: 1101.0822].

[100] Y. -F. Cai, R. Brandenberger and X. Zhang, Preheating a bouncing universe, Phys. Lett. B 703, 25 (2011), [arXiv: 1105.4286].

[101] R. C. Tolman, Relativity, Thermodynamics and Cosmology, Oxford U. Press (1934).

[102] P. J. Steinhardt and N. Turok, Cosmic evolution in a cyclic universe, Phys. Rev. D 65, 126003 (2002), [arXiv: hep-th/0111098].

[103] H. H. Xiong, Y. F. Cai, T. Qiu, Y. S. Piao and X. Zhang, Oscillating universe with quintom matter, Phys. Lett. B 666, 212 (2008), [arXiv:0805.0413].

[104] J. E. Lidsey, D. J. Mulryne, N. J. Nunes and R. Tavakol, Oscillatory universes in loop quantum cosmology and initial conditions for inflation, Phys. Rev. D 70, 063521 (2004), [arXiv: gr-qc/0406042].

[105] Y. S. Piao and Y. Z. Zhang, Inflation in oscillating universe, Nucl. Phys. B 725, 265 (2005), [arXiv: gr-qc/0407027].

[106] Y. -S. Piao, Can the universe experience many cycles with different vacua? Phys. Rev. D 70, 101302 (2004), [arXiv: hep-th/0407258].

[107] H. H. Xiong, T. Qiu, Y. F. Cai and X. Zhang, Cyclic Universe with Quintom matter in Loop Quantum Cosmology, Mod. Phys. Lett. A 24, 1237 (2009), [arXiv:0711.4469].

[108] Y. S. Piao, Proliferation in Cycle, Phys. Lett. B 677, 1 (2009), [arXiv:0901.2644].

[109] Y. -S. Piao, Design of a Cyclic Multiverse, Phys. Lett. B 691, 225 (2010), [arXiv:1001.0631].

[110] Z. -G. Liu and Y. -S. Piao, Scalar Perturbations Through Cycles, [arXiv:1201.1371 ].

[111] S. Mukherji and M. Peloso, Bouncing and cyclic universes from brane models, Phys. Lett. B 547, 297 (2002), [arXiv:hep-th/0205180].

[112] L. Baum and P. H. Frampton, Turnaround in Cyclic Cosmology, Phys. Rev. Lett. 98, 071301 (2007), [arXiv:hep-th/0610213].

[113] V. Sahni and A. Toporensky, Cosmological Hysteresis and the Cyclic Universe, Phys. Rev. D 85, 123542 (2012), [arXiv: 1203.0395].

[114] Y. -F. Cai, E. N. Saridakis, M. R. Setare and J. -Q. Xia, Quintom Cosmology: Theoretical implications and observations, Phys. Rept. 493, 1 (2010), [arXiv:0909.2776].

[115] M. Novello and S. E. P. Bergliaffa, Bouncing Cosmologies, Phys. Rept. 463, 127 (2008), [arXiv:0802.1634].

[116] Y. -F. Cai and E. N. Saridakis, Non-singular Cyclic Cosmology without Phantom Menace, J. 
Cosmol. 17, 7238 (2011), [arXiv: 1108.6052].

[117] N. Arkani-Hamed, H. Georgi and M. D. Schwartz, Effective field theory for massive gravitons and gravity in theory space, Annals Phys. 305, 96 (2003), [arXiv:hep-th/0210184].

[118] L. Grisa and L. Sorbo, Pauli-Fierz Gravitons on Friedmann-Robertson-Walker Background, Phys. Lett. B 686, 273 (2010), [arXiv: 0905.3391]. 